\title{
ON THE CONVEX HULL OF 3-CYCLES OF THE COMPLETE GRAPH
}

\author{
Michel Kovalev \\ Faculty of Applied Mathematics and Informatics \\ University of Belarus \\ Minsk - Belarus \\ Jean-François Maurras * \\ Laboratoire d'informatique fondamentale de Marseille \\ UMR 6166 CNRS \\ Marseille Cedex 9 - France \\ jean-francois.maurras@lim.univ-mrs.fr \\ Yann Vaxès \\ Laboratoire d'informatique fondamentale de Marseille \\ UMR 6166 CNRS \\ Marseille Cedex 9 - France \\ * Corresponding author/autor para quem as correspondências devem ser encaminhadas \\ Recebido em 04/2002, aceito em 11/2002 após 1 revisão
}

\begin{abstract}
Let $K_{n}$ be the complete undirected graph with $n$ vertices. A 3-cycle is a cycle consisting of 3 edges. The 3-cycle polytope is defined as the convex hull of the incidence vectors of all 3-cycles in $K_{n}$. In this paper, we present a polyhedral analysis of the 3-cycle polytope. In particular, we give several classes of facet defining inequalities of this polytope and we prove that the separation problem associated to one of these classes of inequalities is NP-complete. Finally, it is proved that the 3-cycle polytope is a 2-neighborly polytope.
\end{abstract}

Keywords: polytope; cycle; facet; NP-completeness. 


\section{Introduction}

A 3-cycle is a cycle with three edges. Consider the following minimum weighted 3-cycle problem: given a graph $G=(V, E)$ and a 'weight' function $w: E \rightarrow \mathbb{Q}$, find a 3-cycle $C$ of $G$ such that $w(C)$ is as small as possible. This problem can easily be solved in polynomial time by complete enumeration of the triangles $G$.

Let $P(G)$ be the polytope defined as the convex hull of the incidence vectors of the 3-cycles of $G$, that is

$$
P(G)=\operatorname{conv} \cdot h u l l\left\{\chi^{C} \in\{0,1\}: C \text { is a } 3 \text {-cycle of } G\right\} .
$$

The minimum weighted 3-cycle problem is clearly equivalent to the linear program

$$
\max \{w x: x \in P(G)\},
$$

as every minimum weighted 3-cycle yields an optimal vertex solution of the linear program and vice versa. Since the minimum weighted 3-cycle problem is solvable in polynomial time, it follows from the work of Grötschel, Lovász \& Schrijver $(1981,1993)$ that there exists a polynomial time algorithm that solves the following problem:

Separation problem (SEP): given a graph $G=(V, E)$ and a vector $y \in \mathbb{Q}^{E}$, decide whether $y$ belongs to $P(G)$ or not, in the later case, find a vector $a \in \mathbb{Q}^{E}$ such that $a x<$ ay for all $x \in P(G)$.

This algorithm for problem SEP provides an implicit description for $P(G)$. Motivated by the existence of an implicit description for $P(G)$, we attempt to find an explicit description of $P\left(K_{n}\right)$ by a minimal system of linear inequalities. In this paper, we present several classes of facet-defining linear inequalities for $P\left(K_{n}\right)$, we prove that it is NP-hard to solve the separation problem for one of these classes, we show that the diameter of $P\left(K_{n}\right)$ is one. Unfortunately, we did not succeed in our pursuit for a complete description of $P\left(K_{n}\right)$ by a reasonable number of classes of linear inequalities. Using a computer we were able to verify that the facet-defining inequalities presented provide a complete description for $P\left(K_{6}\right)$ (70 facets) and $P\left(K_{7}\right)$ (896 facets). See Barahona \& Grötschel (1986), Coullard \& Pulleyblank (1989) and Seymour (1979) for related studies concerning other cycle polytopes.

Let us introduce some definitions and notations. For a cycle $\mathrm{C}$, define its incidence vector $\chi^{C} \in \mathbb{Q}^{E}$ by letting $\chi_{e}^{C}=1$ if $e \in C$ and 0 otherwise. Throughout this paper, we will confuse a cycle $\mathrm{C}$ with its incidence vector, e.g. we will say that a cycle $\mathrm{C}$ satisfies an inequality. Let $G=(V, E)$ be an undirected graph. For any two adjacent vertices $u$ and $v$, denote by $u v$ the edge between $u$ and $v$. A cycle $C$ of $G$ will be viewed as a set of edges but denoted by an ordered list of vertices; e.g. $\left(v_{1}, v_{2}, v_{3}, v_{4}\right)$ denotes the cycles containing edges $v_{1} v_{2}, v_{2} v_{3}, v_{3} v_{4}, v_{4} v_{1}$. A 3-cycle is a simple cycle of length 3. For two subsets $U$ and $W$ of $V$, we define the subset of edges $(U: W)$ as follows

$$
(U: W):=\{u w \in E: u \in U \text { and } w \in W\}
$$


and $\delta(U):=(U: V-U)$. For a subset $X$ of vertices, let $E(X)$ be the set of edges in $u v$ with $u, v \in X$, and vice versa, for a subset $F$ of edges, let $V(F)$ be the set of end-vertices of edges in $F$. A cycle $C$ is called tight with respect to an inequality $a x \leq b$ if $a \chi^{C}=b$. Finally, for a given subset of edges $F$ and a given vector $x \in \mathbb{R}^{E}$, we adopt the following notation $x(F):=\sum_{e \in F} x_{e}$.

In the next section, we present a few basic properties of $P\left(K_{n}\right)$ and we establish an auxiliary lemma which will be used several times in the rest of the paper for proving that an inequality defines a facet of $P\left(K_{n}\right)$. In Section 3, we provide a complete description of $P\left(K_{n}\right)$ for $n \leq 6$ employing three classes of facet defining inequalities. Then, three new classes of facet defining inequalities are introduced. Altogether, they allows to describe completely $P\left(K_{7}\right)$. We prove that it is NP-hard to solve the separation problem for one of these classes. Next, we present a class of facet defining inequalities that generalizes four classes introduced before and give an additional classes of facets for $P\left(K_{n}\right)$ with $n \geq 9$. Finally, in Section 4 we prove that $P\left(K_{n}\right)$ is a 2-neighborly polytope for all $n \geq 4$.

\section{Basic results}

Let us start with some observations which will be useful later.

Lemma 1. If all 3-cycles of a $K_{4}$ induced by the subset of vertices $\{u, v, w, t\} \subseteq V$ satisfy an equality $a x=b$ then

$$
\begin{gathered}
a_{u v}=a_{w t}=a_{1}, \\
a_{v w}=a_{u t}=a_{2}, \\
a_{u w}=a_{v t}=a_{3}, \\
a_{u v}+a_{v w}+a_{u w}=\beta .
\end{gathered}
$$

Proof. Let us consider all 3-cycles of a $K_{4}$

$$
\begin{gathered}
a_{u v}+a_{v w}+a_{w u}=\beta, \\
a_{v w}+a_{w t}+a_{t v}=\beta, \\
a_{u w}+a_{w t}+a_{t u}=\beta, \\
a_{u v}+a_{v t}+a_{t u}=\beta .
\end{gathered}
$$

Summing up any two of these equalities and subtracting the two others, we get

$$
\begin{aligned}
& a_{u v}-a_{w t}=0, \\
& a_{u w}-a_{v w}=0, \\
& a_{u t}-a_{v w}=0 .
\end{aligned}
$$

$\square$ 
Lemma 2. If all 3-cycles of a $K_{5}$ induced by a subset of vertices $S \subseteq V$ satisfy an equality $a x=b$, then $a_{u v}=\beta / 3$ for all $u, v \in S$.

Proof. Applying Lemma 1 to all 4-cliques defined on $S$, we deduce $a_{u v}=\beta / 3$ for all $u, v \in S$. $\square$

Proposition 1. For $n \geq 5,\left\{x \in \mathbb{Q}^{E}: x(E)=3\right\}$ is the affine hull of $P\left(K_{n}\right)$.

Proof. Suppose that all 3-cycles of $K_{n}$ satisfy an equality $a x=\beta$. By scaling, we may assume that $\beta=3$ and by Lemma $2 a x=\beta$ is precisely $x(E)=3$.

Remark 1. For $n \geq 5$, the dimension of $P\left(K_{n}\right)$ is $\left(\begin{array}{l}n \\ 2\end{array}\right)-1$. For $n=5$, this dimension is 9 . The incidence vectors of the ten 3-cycles of $K_{5}$ are linearly independent. The polytope $P\left(K_{5}\right)$ is a 9-dimensional simplex which is defined $x(E)=3$ and

$$
x(\delta(X)) \leq 2 \text { for each } X \subseteq V,|X|=2 .
$$

Moreover, these inequalities define facets of $P\left(K_{5}\right)$. Indeed, nine of the ten 3-cycles of $K_{5}$ are tight with respect to a given inequality from (1).

\section{Facet defining inequalities}

In the rest of the paper, in order to prove that a valid inequality $I$ defines a facet of $P\left(K_{n}\right)$, we proceed as follows. Consider the linear variety defined by $x(E)=3$ and $I$, if the set of 3 -cycles that are tight with respect to $I$ does not span this variety, then they belong to a proper subvariety, i.e. they satisfy another equality $J \equiv a x=\beta$ such that $I, J$ and $x(E)=3$ are independent. By adding an appropriate linear combination of $x(E)=3$ and $I$ to $J$ we can fix two coefficients of $J$ to 0 . Finally, using the fact that all tight 3-cycles with respect to $I$ satisfy $J$ we derive that $a_{e}=\beta=0$ for all $e \in E$.

Proposition 2. For each edge $u v \in E$, the linear inequality

$$
x_{e} \geq 0
$$

defines a facet of $P\left(K_{n}\right)$ whenever $n \geq 6$.

Proof. Suppose that all tight 3-cycles with respect to (2) (that is, all 3-cycles not containing the edge $u v$ ) satisfy an inequality $a x=\beta$. Applying Lemma 2 to all $K_{5}$ not containing the edge $u v$ we deduce that $a_{e}=\beta / 3$ for all $e \in E-\{u v\}$. Finally, fixing $\beta=0$ and $a_{u v}=0$ we get $a_{e}=\beta=0$ for all $e \in E$. 


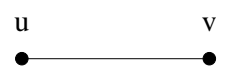

(2)

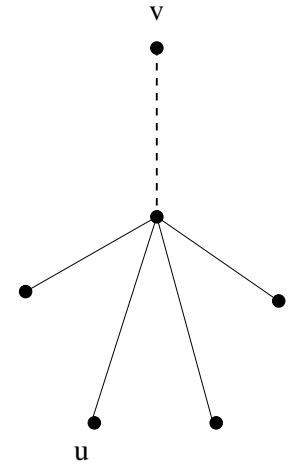

(3)

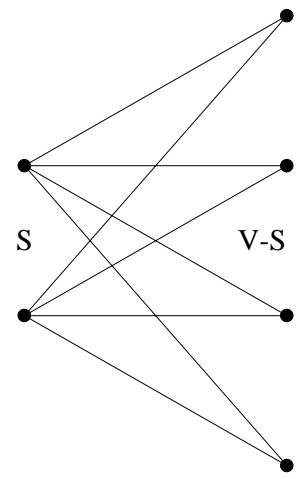

(4)

Lemma 3. Let $u, v \in V$ and $n \geq 6$ if all tight with respect to

$$
x(\delta(u)-u v)-x_{u v} \geq 0
$$

3-cycles of $K_{n}$ satisfy ax $=\beta$ then

$$
\begin{array}{ll}
a_{u v}=a_{1}, & \\
a_{v w}=2 \beta / 3-a_{1}=a_{2}, & \text { for all } w \in V-\{u, v\}, \\
a_{e}=\beta / 3=a_{3}, & \text { for all } e \in E-\delta(u) .
\end{array}
$$

Proof. The 3-cycles of $K_{n-1}$ not containing $u$ are tight with respect to (3), thus they satisfy $a x=\beta$. Using Lemma 2 we derive $a_{e}=\beta / 3=a_{3}$ for all $e \in E-\delta(u)$. Now, all 3-cycles $(u, v, w)$ with $w \in V-\{u, v\}$ are tight with respect to (3) yielding $a_{u w}=2 \beta / 3-a_{u v}$ for all $w \in V-\{u, v\}$.

Proposition 3. For each edge $u v \in E$, the inequality (3) defines a facet of $K_{n}$ whenever $n \geq 6$.

Proof. First apply Lemma 3, then fix two coefficients $\beta=a_{1}=0$, yielding $a_{2}=a_{3}=0$. $\square$

The set of all integer solutions of the system $x(E)=3,(2)$ and (3) is exactly the set of all 3-cycles of $K_{n}$.

Proposition 4. For each subset $X \subseteq V$ such that $2 \leq|X| \leq|V| / 2$, the inequality

$$
x(\delta(X)) \leq 2
$$

defines a facet of $P\left(K_{n}\right)$ whenever $n \geq 6$. 
Proof. Let us suppose that all tight 3-cycles with respect (4) belong to a proper subvariety defined by $x(E)=3$, (4) and $a x=\beta$. Note that all 3-cycles of a $K_{4}$ containing two vertices $u, v \in X$ and two other $u, v \in V-X$ are tight with respect to (4). Applying Lemma 1 to these $K_{4}$, we obtain

$$
\begin{array}{ll}
a_{u v}=a_{w t}=a_{1} & \text { for all } u, v \in X \text { and } w, t \in V-X \\
a_{u w}=a_{2} & \text { for all } u \in X \text { and } w \in V-X
\end{array}
$$

with $a_{1}+2 a_{2}=\beta$. By fixing $a_{1}=a_{2}=0$, we get $\beta=0$ and $a_{e}=0$, for all edge $e \in E$.

Using a computer code, we have been able to enumerate all facets of $P\left(K_{6}\right)$. This polytope has 70 facets and is completely defined by inequalities (2), (3), (4) and $x(E)=3$.

Proposition 5. Let $G=(V, E)$ be a graph and let

$$
Q(G)=\left\{x \in \mathbb{R}^{E}: x(\delta(X)) \leq 2 \text { for all } X \subseteq V, 2 \leq|X| \leq|V|-2\right\} .
$$

The separation problem for $Q(G)$ is NP-complete.

Proof. We provide a polynomial reduction from the problem MAXCUT which is proved to be NP-hard (Garey, Johnson \& Stockmeyer, 1976). Its formulation follows. Given an undirected graph $H=(V, F)$ and a positive integer $k$, find a subset of vertices $X \subseteq V$ such that $|\delta(X)|>k$. One can transform an instance of the MAXCUT problem in an instance of the separation problem for $Q\left(K_{n}\right)$ as follows. Suppose without loss of generality. that no vertex of $H$ has a degree larger than $k$ (otherwise one can find a cut of cardinality larger than $\mathrm{k}$ in linear time). Then, consider a real valued vector $x \in \mathbb{R}^{E}$ defined as follows

$$
x_{e}=\left\{\begin{array}{l}
2 / k \text { if } e \in F \\
0 \text { if } e \in E-F
\end{array}\right.
$$

Clearly, there is a subset $X \subseteq V$ such that (4) separates $x$ from $Q\left(K_{n}\right)$ if and only if there is a cut of cardinality larger than $k$ in $H$. This concludes the proof of Proposition 5.

Proposition 6. For each subset of four vertices $\{u, v, w, t\} \subseteq V$, the inequality

$$
x(\delta(u)-\{u v, u w\})-x_{u v}+x(\delta(w)-\{w t, u w\})-x_{w t} \geq 0
$$

defines a facet of $P\left(K_{n}\right)$ whenever $n \geq 7$.

Proof. Consider the complete subgraph $K_{n-1}$ which does not contain the vertex $v$. Since it has at least 6 vertices, as in the proof of Proposition 3, we can show that $a_{e}=0$ for each edge $e$ of this subgraph, and thus $\beta=0$. Analogously, one can show that the same equality holds for all edges of the subgraph $K_{n-1}$ which does not contain $w$. It remains to fix $a_{u w}$. Note that $\beta=0$ and consider one of the two 3-cycles containing the edge $v w$ and which is tight with respect to (5), namely $(u, v, w)$ or $(u, t, w)$. We obtain $a_{u w}=0$. $\square$ 


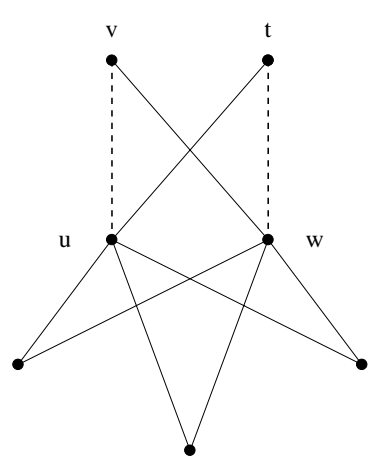

(5)

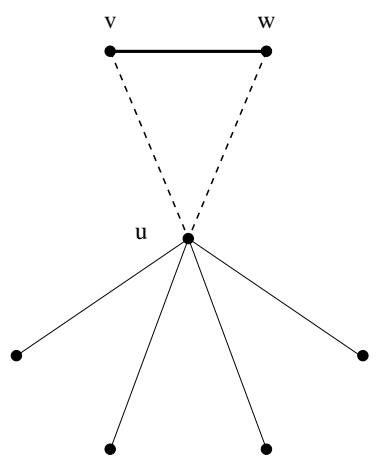

(6)

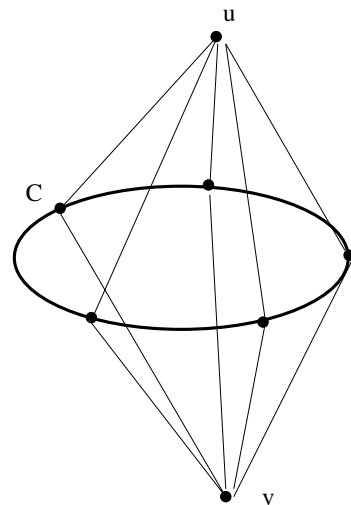

(7)

Proposition 7. For each subset of three vertices $\{u, v, w\} \subseteq V$, the inequality

$$
x(\delta(u)-\{u v, u w\})+2 x_{v w}-x_{u v}-x_{u w} \geq 0
$$

defines a facet of $K_{n}$ whenever $n \geq 7$.

Proof. The proof is similar to that of Proposition 3. First, consider the complete subgraph $K_{n-1}$ which does not contain the vertex $v$ and then the one which does not contain $w$. Finally, consider the 3-cycle $(u, v, w)$ which is tight with respect to inequality (6) and contains the edge $v w$. $\square$

Proposition 8. For a pair of vertices $\{u, v\} \subseteq V$, and each simple cycle $C$ containing all vertices of $V-\{u, v\}$ the inequality

$$
x(\delta(u)-\{u v\})+x(\delta(v)-\{u v\})+2 x(C) \geq 2
$$

defines a facet of $P\left(K_{7}\right)$.

Proof. Consider a $K_{4}$ induced by $u, v$ and any two non consecutive vertices $w$ and $t$ of the cycle C. Using Lemma 1 we derive $a_{w t}=a_{u v}=a_{1}$ and $a_{u w}=a_{v t}=a_{2}$ for each $v, w \in C$. It remains to fix the coefficients of the edges of the cycle $C$. Let us consider a 3 -cycle $(v, w, t)$ which contains only one edge $w t$ of $C$. This 3-cycle is tight with respect to (7) implying $a_{w t}=\beta-2 a_{1}$. Finally, we fix $a_{1}=a_{2}=0$. and by considering a tight 3 -cycle $(u, v, w)$, we deduce $\beta=0$ and $a_{e}=0$ for each edge $e \in C$.

Using a computer code, we have been able to enumerate all 896 facets of $P\left(K_{7}\right)$. This polytope is completely defined by inequalities (2)-(7) and equality $x(E)=3$. Note that six classes of inequalities are necessary to describe completely $P\left(K_{7}\right)$. Note that, for $n \geq 8$, the inequality (7) is not valid since it is violated by any 3 -cycle consisting of vertices of $C$ and not containing any edge of $C$. 


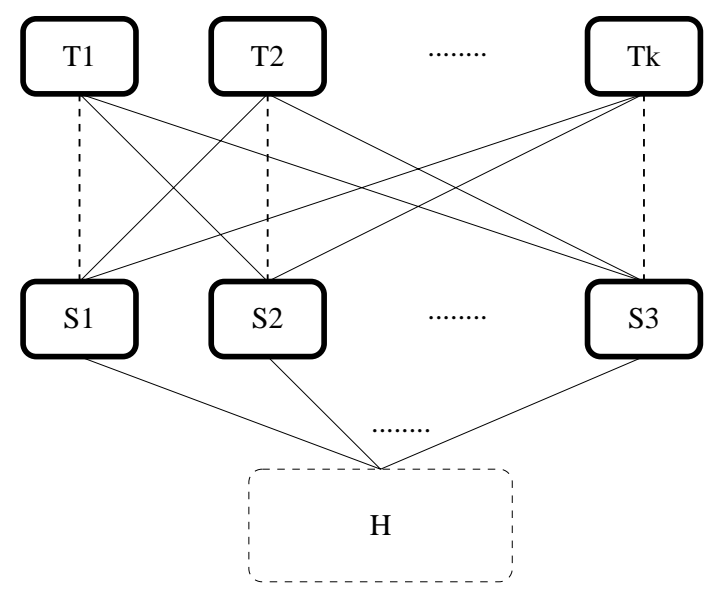

Now, we present a class of facet defining inequalities that generalizes the classes (3), (5) and (6). Given a positive integer $k$, a list $S_{i}, T_{i},(i=1, \ldots, k)$ of disjoint subsets of $V$, we define the following subsets of edges

$$
\begin{aligned}
& H=V-\bigcup_{i=1}^{k} S_{i} \cup T_{i}, E_{S, T}=\bigcup_{i}\left(S_{i}: T_{i}\right), E_{\overline{S, T}}=\bigcup_{i \neq j}\left(S_{i}: T_{j}\right), \\
& E_{S, S}=\bigcup_{i}\left(S_{i}: S_{i}\right), E_{T, T}=\bigcup_{i}\left(T_{i}: T_{i}\right), E_{H, S}=\bigcup_{i}\left(T_{i}: T_{i}\right) .
\end{aligned}
$$

Consider the following inequality

$$
2\left(x\left(E_{S, S}\right)+x\left(E_{T, T}\right)\right)-x\left(E_{S, T}\right)+x\left(E_{\overline{S, T}}\right)+x\left(E_{H, S}\right) \geq 0
$$

Proposition 9. If $n \geq 5,|H|+k \geq 5$, and at least one of the following conditions holds:
1. $k \geq 2$
2. $\left|\mathrm{S}_{1}\right|=1$
3. $\left|\mathrm{T}_{1}\right| \geq 2$

then (8) defines a facet of $P\left(K_{n}\right)$.

Proof. We distinguish two cases.

Case 1: $k \geq 2$.

Consider one after another all $K_{4}$ obtained by picking a vertex in each subset $S_{i}, T_{i}, S_{j}$ and $T_{j}$ for $i, j=1, \ldots, k, i \neq j$. By applying Lemma 1 for each of these $K_{4}$ we get the following equalities

$$
\begin{array}{ll}
a_{s_{i} t_{j}}=a_{1} & \text { for each } s_{i} \in S_{i}, t_{i} \in T_{i} \\
a_{s_{i} t_{j}}=a_{2} & \text { for each } s_{i} \in S_{i}, t_{j} \in T_{j} \text { with } i \neq j \\
a_{s_{i} s_{j}}=a_{t_{i} t_{j}}=a_{3} & \text { for each } s_{i} \in S_{i}, s_{j} \in S_{j}, t_{i} \in T_{i}, t_{j} \in T_{j} \text { with } i \neq j \\
a_{1}+a_{2}+a_{3}=\beta &
\end{array}
$$


Let $\left\{s_{1}, \ldots, s_{k}\right\}$ and $\left\{t_{1}, \ldots, t_{k}\right\}$ be two subsets of vertices such that $s_{j} \in T_{j}$ and $t_{j} \in T_{j}$ for all $j=1, \ldots, k$. For $i=1, \ldots, k$, define $P_{i}:=H \cup\left\{s_{i}\right\} \cup\left\{t_{1}, t_{2}, \ldots, t_{k}\right\}$. By hypothesis $\left|P_{i}\right|=|H|+k+1 \geq 6$, hence by applying Lemma 3 to the complete subgraph induced by $P_{i}$ we derive

$$
\begin{array}{ll}
a_{s_{i} v}=a_{2}=2 \beta / 3-a_{1} & v \in H \\
a_{v w}=a_{t_{j} v}=a_{t_{j} t_{l}}=a_{3} & j, l \in\{1, \ldots, k\}, j \neq l, v, w \in H .
\end{array}
$$

Note that any 3-cycle with two vertices in $T_{i}$ and one in $S_{i}$ (or the reverse) satisfy (8), therefore

$$
a_{r_{i} s_{i}}=a_{q_{i} t_{i}}=\beta-2 a_{1} \quad \text { for each } i=1, \ldots, k, r_{i}, s_{i} \in S_{i}, q_{i}, t_{i} \in T_{i} .
$$

Fixing two coefficients $\beta=a_{1}=0$, we get $a_{2}=a_{3}=a_{4}=0$. This concludes Case 1 .

Case 2: $k=1$. We distinguish two subcases.

$\left|S_{l}\right|=1$.

If $\left|T_{1}\right|=1$ then the proof of Proposition 3 applies. Otherwise, let $t_{1}, q_{1}$ be two vertices in $T_{l}$. We can apply Lemma 1 on the subgraphs induced respectively by the subsets of vertices $P_{l}=\left\{s_{l}\right\} \cup\left\{t_{l}\right\} \cup H$ and $Q_{l}=\left\{s_{l}\right\} \cup\left\{q_{l}\right\} \cup H$ and derive

$$
\begin{array}{rr}
a_{s_{1} t_{1}}=a_{1} & \\
a_{s_{1} v}=2 \beta / 3-a_{1}=a_{2} \quad \text { for each } v \in H \\
a_{v w}=a_{t_{1} v}=\beta / 3=a_{3} & \text { for each } v, w \in H \\
\text { and } & \\
a_{s_{1} q_{1}}=a_{1}^{\prime} \quad \text { for each } v \in H \\
a_{s_{1} v}=2 \beta / 3-a_{1}^{\prime}=a_{2}^{\prime} \quad \text { for each } v, w \in H \\
a_{v w}=a_{q_{1} v}=\beta / 3=a_{3}^{\prime}
\end{array}
$$

Hence, $a_{2}^{\prime}=a_{2}, a_{3}^{\prime}=a_{3}$, and we deduce that $a_{1}^{\prime}=a_{1}$. These equalities do not depend on the choice of the vertices $t_{l}, q_{l}$. Finally, note that all 3-cycles having two vertices in $T_{l}$ and one in $S_{I}$ satisfy (8), hence

$$
a_{t_{1} q_{1}}=\beta-2 a_{1}=a_{4} \text { for each } t_{1}, q_{1} \in T_{1}
$$

By fixing $\beta=a_{1}=0$, we get $a_{2}=a_{3}=a_{4}=0$.

$\left|S_{l}\right| \geq 2$.

In this subcase, $\left|T_{1}\right| \geq 2$ because one of the three conditions of the proposition must hold. Choose two vertices $s_{1}, s_{1}{ }_{1} \in S_{1}$. For each of them we can provide the same proof as in the case $\left|S_{l}\right|=1$ and show that 


$$
\begin{array}{rlr}
a_{s_{1} t_{1}} & =a_{1} & \text { for each } t_{1} \in T_{1} \\
a_{s_{1} v}=2 \beta / 3-a_{1}=a_{2} & \text { for each } v \in H \\
a_{v w}=a_{t_{1} v}=\beta / 3=a_{3} & \text { for each } v, w \in H \\
a_{t_{1} q_{1}}=\beta-2 a_{1}=a_{4} & \text { for each } t_{1}, q_{1} \in T_{1} \\
\text { and } & \\
a_{s_{1} q_{1}}=a_{1}^{\prime} & \text { for each } t_{1} \in T_{1} \\
a_{s_{1}^{\prime} v}=2 \beta / 3-a_{1}^{\prime}=a_{2}^{\prime} & \text { for each } v \in H \\
a_{v w}=a_{q_{1} v}=\beta / 3=a_{3}^{\prime} & \text { for each } v, w \in H, t_{1} \in T_{1} \\
a_{t_{1} q_{1}}=\beta-2 a_{1}^{\prime}=a_{4}^{\prime} & \text { for each } t_{1}, q_{1} \in T_{1}
\end{array}
$$

We get $a_{3}^{\prime}=a_{3}$ and $a_{4}^{\prime}=a_{4}$, and deduce $a_{1}^{\prime}=a_{1}$ and $a_{2}^{\prime}=a_{2}$. Fixing $\beta=a_{1}=0$, we concludes $a_{2}=a_{3}=a_{4}=0$.

Proposition 10. Let $C$ and $C^{\prime}$ be two simple cycles covering all vertices of $K_{n}$ and such that if $u v$ and $v w$ belongs to $C$, then uw belong to $C^{\prime}$. The inequality

$$
x(C)-x\left(C^{\prime}\right) \leq 1
$$

defines a facet of $P\left(K_{n}\right)$ whenever $n$ is odd and $n \geq 9$.

Proof. Let $e=u v, e^{\prime}=w t \in C$ be two edges such that the $K_{4}$ induced by the subset of vertices $\{u, v, w, t\}$ has only the edges $u v$ and $w t$ in common with $C$ and $C^{\prime}$. Every 3 -cycles of this $\mathrm{K}_{4}$ are tight with respect to inequality (9). Using Lemma 1 for every such $K_{4}$, we show that

$$
\begin{array}{rr}
a_{e}=a_{1} & \text { for each } e \in C \\
a_{e}=a_{2} & \text { for each } e \in E-\left\{C \cup C^{\prime}\right\} \\
a_{1}+2 a_{2} & =\beta .
\end{array}
$$

Next, consider the 3-cycles $\left(e, e^{\prime}, e^{\prime \prime}\right)$ with $e, e^{\prime} \in C$ and $e^{\prime \prime} \in C^{\prime}$. They are also tight with respect to inequality (9), yielding that $a_{e^{\prime \prime}}+2 a_{2}=\beta$ and $a_{e^{\prime \prime}}=a_{3}$. Hence, the following equalities holds

$$
a_{1}+2 a_{2}=\beta \text { and } a_{3}+2 a_{1}=\beta
$$

Finally, we fix $\mathrm{a}_{1}=\mathrm{a}_{2}=0$, and concludes $\beta=\mathrm{a}_{3}=0$.

\section{Neighbourhood relation on $P\left(K_{n}\right)$}

A polyhedron $P$ is said to be $k$-neighborly if each $k$-subset $S \subseteq \operatorname{vert}(P)$ defines a face $F=\operatorname{conv}(S)$ such that $S=\operatorname{vert}(F)$.

Proposition 11. $P\left(K_{n}\right)$ is a 2-neighborly polytope whenever $n \geq 4$.

Proof. Given any two 3-cycles $x=\left(v_{1} v_{2} v_{3}\right)$ and $x^{\prime}=\left(v^{\prime}{ }_{1} v_{2}{ }_{2} v_{3}{ }_{3}\right)$, the incidence vector of the subgraph obtained as the union of $x^{\prime}$ and $x^{\prime \prime}$ cannot be written as a convex linear combination of any other 3 -cycles. Therefore, the intersection of $\operatorname{conv}\left(\operatorname{vert}\left(P\left(K_{n}\right)-\left\{x^{\prime}, x^{\prime \prime}\right\}\right)\right)$ and $\operatorname{aff}\left(\left\{x^{\prime}, x^{\prime \prime}\right\}\right)$ is empty. In other words, $\operatorname{conv}\left(\left\{x^{\prime}, x^{\prime \prime}\right\}\right)$ is a 1 -face (an edge) of $P\left(K_{n}\right)$. 
Following Grünbaum (1967), we conclude that each 3-face of $P\left(K_{n}\right)$ is a simplex, the diameter of $P\left(K_{n}\right)$ is equal to 1 , and the number of 1-faces of $P\left(K_{n}\right)$ is equal to $\left(\begin{array}{c}n(n-1)(n-2) / 6 \\ 2\end{array}\right)$

Furthermore, notice that for a linear program over $P\left(K_{n}\right)$, the problem of finding the best neighbour of an extreme point is equivalent to the complete enumeration.

\section{Acknowledgement}

We would like to thank anonymous referees for many helpful comments.

\section{References}

(1) Barahona, F. \& Grötschel, M. (1986). On the cycle polytope of a binary matroid. Journal of Combinatorial Theory Ser. B, 40-62.

(2) Coullard, C.R. \& Pulleyblank, W.R. (1989). On cycles cones and polyhedra. Linear Algebra and Its Applications, 114/115, 613-640.

(3) Garey, M.R.; Johnson, D.S. \& Stockmeyer, L. (1976). Some Simplified NP-Complete Problems. Theoretical Computer Science, 1, 237-267.

(4) Grötschel, M.; Lovász, L. \& Schrijver, A. (1981). The ellipsoid method and its consequences in combinatorial optimization. Combinatorica, 2, 169-197 [Corrigendum: Combinatorica (1984), 4, 291-295].

(5) Grötschel, M.; Lovász, L. \& Schrijver, A. (1993). Geometric algorithms and combinatorial optimization. Algorithms and Combinatorics, 2, Springer-Verlag, Berlin.

(6) Grünbaum, B. (1967). Convex Polytopes. Interscience Publisher, John Wiley \& Sons.

(7) Seymour, P.D. (1979). Sums of circuits. In: Graph Theory and Related Topics [edited by J.A. Bondy and U.S.R. Murty], Academic Press, New York, 341-355. 